\title{
ARTICLE
}

\section{Assessing and managing hallucinations in children and adolescents}

\author{
Maria-de-Gracia Dominguez \& M. Elena Garralda
}

\begin{abstract}
Maria-de-Gracia Dominguez is an honorary clinical lecturer in child and adolescent psychiatry at Imperial College London, and works as a consultant child and adolescent psychiatrist with Central and North West London (CNWL) NHS Foundation Trust. Her $\mathrm{PhD}$ research investigated the dynamic process driving psychotic expression from mental wellness to the onset of clinical psychosis in young people from the general population. Her research interest expanded to investigate the developmental expression of psychosis in clinical samples of adolescents, as an age group associated with increased risk for the emergence of psychosis. Elena Garralda is Emeritus Professor of Child and Adolescent Psychiatry and an honorary consultant with CNWL NHS Foundation Trust. Her research interests include the interface between physical and mental problems and psychotic symptoms in children and young people. Correspondence Dr Mariade-Gracia Dominguez, Centre for Mental Health, Imperial College London, Hammersmith Hospital Campus, 7th Floor Commonwealth Building, Du Cane Road, London W12 ONN, UK. Email: m.dominquezbarrera@imperial.ac.uk
\end{abstract}

\begin{abstract}
SUMMARY
Hallucinations (erroneous percepts in the absence of identifiable stimuli) are a key feature of psychotic states, but they have long been known to present in children with non-psychotic psychiatric disorders. Recent epidemiological studies of child populations found surprisingly high rates (about $10 \%$ ) of hallucinatory experiences. These hallucinatory phenomena are most likely to occur in the absence of psychiatric disorder and are usually simpler, less elaborate and less distressing than those observed in children with psychiatric disorders. This article details the clinical assessment of hallucinations in children and adolescents, taking into account developmental considerations and paediatric organic associations. It describes hallucinations in young people with psychoses (schizophrenia spectrum and mood disorders) and non-psychotic psychiatric disorders (emotional and behavioural disorders), and it addresses therapeutic aspects.
\end{abstract}

\section{LEARNING OBJECTIVES}

- Be aware of the frequency, associations and clinical significance of hallucinatory experiences in general populations of children and young people

- Know how to conduct clinical assessments of children and young people presenting with hallucinatory experiences, taking into account developmental considerations

- Be able to recognise the characteristics and clinical associations of hallucinations in children with psychotic and/or non-psychotic disorders and understand the therapeutic implications

\section{DECLARATION OF INTEREST}

None

From the earliest stages of life, the experiences of smelling, touching, tasting, hearing and seeing are the front door through which babies relate to the world that surrounds them. Sensations are interpreted as perceptions and processed into inner representations mediated by complex cognitive processing in the brain, involving the optimal combination of new sensory inputs with prior knowledge (Jardri 2014). Human perception can be distorted in different ways. Taking into account the presence or absence of external stimuli, and the level of consciousness, a variety of perception distortions are defined (Table 1) (Casey 2007).

Hallucinations refer to sensory experiences that occur: (i) in the absence of corresponding external stimulation of the relevant sensory organ, (ii) in the awake state and (iii) with a sufficient sense of reality to resemble a veridical perception over which the individual does not feel that they have direct and voluntary control. Hallucinations have been considered one of the paradigmatic psychotic symptoms, together with their counterpart, alterations in thought content or delusions. Nonetheless, in both adult and child samples hallucinations are present not only in psychotic disorders such as schizophrenia spectrum or mood disorders, but also in other psychiatric disorders, including anxiety, stress reactions and conduct disorders, in substance misuse and medical conditions such as epilepsy and delirium, as well as in mentally healthy people.

\section{Epidemiological findings}

Over recent years it has become evident that hallucinatory experiences are common in general adult and child populations. Following the hypothesis of the continuum of psychopathology and medical help-seeking, psychopathological features would be present in the general population, although in an attenuated and/or isolated form and with lower levels of distress and impairment not leading to need for care. Epidemiological studies have explored their presence through questions that identify both clinical hallucinations (that fulfil the above criteria for the definition of hallucinations and cause distress and functional impairment significant enough to lead to helpseeking) and broader hallucinatory experiences (that do not cause sufficient distress or impairment to lead to help-seeking). Epidemiological questionnaires have used questions such as 'Have you ever heard voices or sounds that no one else 
can hear?' or 'Have you ever heard voices when you were alone?', which target a continuum of experience from single noises or words more in line with hallucinatory experiences to clinically relevant ongoing conversing voices. Overall, about $10 \%$ of children and young people in the general population report some type of hallucinatory experience (Linscott 2013; Jardri 2014).

\section{Hallucinatory experiences and age}

Although evidence on the associations between age and hallucinatory experiences is not unequivocal, recent studies on the trajectory of hallucinatory experiences over time have found higher prevalence rates in childhood (13-17\% at age 9-12) and a decrease in adolescence (5-7.5\% at age 13-18) (Kelleher 2012a; Thapar 2012). However, it is of note that, whereas psychotic experiences may be highly reported by children, the prevalence of diagnosed psychosis conversely rises steeply in adolescence. This indicates that psychotic experiences are only rarely an expression of a psychotic disorder in children, and it further suggests either that hallucinatory experiences are part of normal developmental trajectories for a number of children or - as discussed later - that the high general population rates reflect difficulty in identifying hallucinations accurately in young children.

\section{Hallucinatory experiences, emotional and behavioural symptoms}

Cross-sectional studies have documented positive associations between hallucinatory experiences and a range of concurrent emotional and behavioural symptoms and disorders in children and young people (Jardri 2014). As may be expected, professional help-seeking in children with abnormal auditory experiences is related to hallucination-related distress, including more emotional triggers of the voices, negative views towards them, and perceiving them as influencing their emotions and behaviour (de Leede-Smith 2013). Associations have also been reported between hallucinatory experiences, traumas such as bullying and suicidal symptomatology (Jardri 2014). This work, however, has rarely controlled for the possible confounding effects of underlying or mediating neurodevelopmental and/or social anomalies, or the severity of the depressive symptoms; the significance of these associations thus requires further investigation.

\section{Persistence of hallucinatory experiences}

The majority of hallucinatory experiences are transitory (75-90\%) and resolve spontaneously

\section{TABLE 1 Psychopathology of human perception: sensory distortions}

\begin{tabular}{|c|c|}
\hline \multicolumn{2}{|c|}{ In the presence of real external stimuli } \\
\hline \multirow[t]{2}{*}{ Quantitative variation } & $\begin{array}{l}\text { Hypoaesthesia or hyperaesthesia - the involuntary experience of reduced } \\
\text { or amplified intensity in the sensory perception (in any of the five sensory } \\
\text { modalities) of real external stimuli; e.g. in neurological disorders such as } \\
\text { cutaneous sensory disorder or herpes zoster, as well as in manic states, } \\
\text { drug misuse or anxiety. }\end{array}$ \\
\hline & $\begin{array}{l}\text { Metamorphopsia - the involuntary experience of variation in size or shape } \\
\text { of real external stimuli; e.g. in ophthalmological disorders such as macular } \\
\text { degeneration. }\end{array}$ \\
\hline \multirow[t]{2}{*}{ Qualitative variation } & $\begin{array}{l}\text { Derealisation - the involuntary alteration of perception (and attributed } \\
\text { meaning) of the external environment so that it seems unreal; e.g. in } \\
\text { anxiety disorders or epilepsy, jamais vu or déjà vu. }\end{array}$ \\
\hline & $\begin{array}{l}\text { Illusions - the experience of erroneous (auditory or visual) percepts } \\
\text { in the presence of identifiable external stimuli which may lead to } \\
\text { misinterpretation of true sensations; voluntary or involuntary; facilitated } \\
\text { by crepuscular states, fatigue, stress, increased or reduced light } \\
\text { stimulation, noise or affective states. }\end{array}$ \\
\hline \multicolumn{2}{|c|}{ In the absence of real external stimuli } \\
\hline \multirow[t]{3}{*}{$\begin{array}{l}\text { Reduced level of } \\
\text { consciousness }\end{array}$} & $\begin{array}{l}\text { Hypnagogic hallucinations - involuntary erroneous percepts immediately } \\
\text { before falling asleep. }\end{array}$ \\
\hline & $\begin{array}{l}\text { Hypnopompic hallucinations - involuntary erroneous percepts during the } \\
\text { transition from sleep to wakefulness. }\end{array}$ \\
\hline & $\begin{array}{l}\text { Hallucinosis (or hallucinations with decreased consciousness levels) - the } \\
\text { involuntary experience of erroneous percepts in the absence of external } \\
\text { stimuli in compromised level of consciousness; e.g. in alcohol intoxication } \\
\text { or withdrawal, delirium, drug intoxication. }\end{array}$ \\
\hline \multirow[t]{2}{*}{$\begin{array}{l}\text { Normal level of } \\
\text { consciousness }\end{array}$} & $\begin{array}{l}\text { Eidetic images - the experience of vivid visual perceptions that resemble } \\
\text { those previously seen in the external environment or imagined; the image } \\
\text { is not subject to voluntary control or recall; can last for minutes or hours. }\end{array}$ \\
\hline & $\begin{array}{l}\text { Hallucinations - the involuntary experience of erroneous percepts in the } \\
\text { absence of identifiable external stimuli. }\end{array}$ \\
\hline
\end{tabular}

Based on Casey \& Brendan (2007).

over time (Linscott 2013). Traumatic events such as maltreatment, assault or bullying have been identified not only as potential triggers of hallucinatory experiences, but also as risk markers for their persistence (Linscott 2013; Jardri 2014). In addition, persistence has been shown to be predicted in some studies by: (i) higher severity, frequency and complexity of the perceptual phenomena (e.g. multiple voices with external attributions); (ii) the presence of emotional and behavioural symptoms and of drug use; and (iii) other risk factors, including adverse backgrounds, disturbed childhood development, low academic achievement and urbanicity, although these could be mediated, in part at least, by concurrent psychiatric disorders (Dominguez 2011; van Rossum 2011; Thapar 2012; Linscott 2013; Jardri 2014).

\section{Hallucinatory experiences and psychoses}

The great majority of children reporting hallucinatory experiences do not have psychotic states, nor do they ever make the transition to a psychotic disorder. Earlier longitudinal studies showed an increased risk for schizophreniform diagnoses in adulthood in $25 \%$ of children reporting a combination of hallucinatory experiences and delusional 
thoughts aged 11. Later meta-analytic studies have estimated that only about $7 \%$ of children reporting hallucinatory experiences will suffer from a psychotic disorder later in life (Linscott 2013). The positive predictive value of the presence of psychotic-like symptoms is consequently low and of limited use for psychosis prevention.

Even in young people with more troublesome psychotic symptoms - some of whom might qualify as having one of the 'at-risk mental states' - the risk of conversion to psychosis has been estimated as 26\% (16\% converting to schizophrenia) (FusarPoli 2013). Operationalised criteria developed to identify at-risk mental states usually require: attenuated positive psychotic symptoms; or fullblown psychotic symptoms although brief and selflimiting; or a significant decrease in functioning in the context of a genetic risk for schizophrenia; or basic symptoms together with distress, dysfunction and help-seeking (Fusar-Poli 2013).

It seems plausible that the predictive value of childhood hallucinatory experiences for adult psychoses will be accounted for by the experience of complex hallucinatory phenomena in the context of a summation of psychotic indicators, such as delusional beliefs, negative and disorganised symptoms, neurocognitive and social impairment, and behaviour and mood regulation anomalies, in combination with exposure to sufficient environmental stressors (Dominguez 2010; Smeets 2012; Linscott 2013).

\section{A developmental approach to hallucinations}

Hallucinations are subjective phenomena and their assessment in children relies on the child's ability to understand and explain to others the difference between perceptions of externally and internally generated stimuli, and between those that are sleep-related or experienced in full awareness. At what age are children able to do this? In addition, young children have a rich fantasy life: when do they become able to differentiate this from sensory perceptions?

\section{Fantasy and hallucinations}

It has long been established that young children are able to differentiate between fantasy and reality. Despert (1948) studied the behaviour and communications during play of children aged 2-5 and showed that, although fantasy activities were not uncommon, hallucinations were absent. Some children talked about their fantasies repeatedly, but these lacked the characteristics of perceptions and those with the most creative imaginations were readily able to acknowledge their fantasies as 'pretend' rather than reality. This suggested that very young children are already able to appreciate when experiences are generated by them as fantasy and when they are not.

Imaginary friends are an example of fantasy activity by children. They have been reported in nearly half $(46 \%)$ of 5 - to 12 -year-old children (Pearson 2001) and tend to disappear in middle childhood. They have been shown to be associated with positive developmental outcomes, such as superior performance on theory of mind tasks. From a clinical perspective, imaginary companions - in addition to lacking the characteristics of perceptions of seeing and hearing - differ from hallucinations in at least two respects: first, they can often be invoked by the child at will, in contrast to the involuntary nature of hallucinations, and second, they typically function as play partners associated with positive emotions.

\section{Hallucinations in young children}

In clinical samples, hallucinations seem to be especially rare under 7 years of age (Garralda 1984a). This has led to the suggestion that, in young children, limitations in cognitive development militate against the effective communication of hallucinations to others. They would have difficulty not in differentiating selfinduced fantasies or 'pretend' activities from reality, but in knowing the difference between dreams and other subjective phenomena such as hallucinations. In Despert's study (Despert 1948), some 2- to 5-year-old children related hypnagogic experiences, but it proved impossible to obtain sufficient evidence from the children themselves to conclude that the experiences were hypnagogic hallucinations as opposed to dreams. Piaget (1932) pointed out that until 7-8 years of age dreams are still systematically considered as objective reality, 'as a sort of ethereal, rarefied picture floating in the air and fixed before our eyes' (1997 reprint: p. 187). The comparatively high rates of hallucinations reported in epidemiological samples of schoolchildren in response to standardised questioning may therefore be partly an artefact of immature cognition in the younger among them.

Preschool children are more likely to demonstrate the presence of hallucinations through their behaviour - as outlined later - and to be particularly vulnerable to experiencing hallucinations and other abnormal phenomena in conditions of brain toxicity such as high fever and septic illness. In a sample of children and adolescents with meningococcal disease, 'out-ofbody' type experiences were mainly reported by preschool children, possibly reflecting cognitive or 
brain immaturity through failure by the brain to integrate complex somatosensory and vestibular information (Shears 2005).

\section{Assessing hallucinations in clinical samples}

When a child presents with hallucinatory experiences, the first step is to clarify whether these are:

- illusions or misrepresentations of sensory inputs, fantasy-related, as with imaginary friends ('Can you bring them on? Do you actually see them and hear them as you see and hear me now?')

- intrusive thoughts or inner images, as in obsessions, where ego-dystonic experiences are personalised and attributed to an external source ("Are they like the voices you normally hear through your ears, or are they more like thoughts as when you are thinking about things?')

- post-traumatic flashbacks, as in involuntary and intrusive vivid inner images or sounds from past traumatic memories.

Once the presence of hallucinations is established, the second task is to document their complexity: simple hallucinations such as occasionally hearing one's own name being called or fleetingly seeing shadows out of the corner of one's eye are regarded as non-clinically significant.

Third, the clinician needs to ascertain whether hallucinations are linked to states of lowered awareness, as in sleep (hypnagogic or hypnopompic), fever, drug-taking, toxicity, epilepsy or migraine. Alice in Wonderland syndrome presents as a combination of migraine and a variety of hallucinations and illusions of size, time, colour, body shape and movement (Smith 2015). The differentiation of sleep-related phenomena and hallucinations merits particular note (Table 1). Although hypnagogic and hypnopompic hallucinations are usually without clinical significance, there are specific cases in which they may be part of a disabling childhood sleep disorder such as narcolepsy.

Finally, the fourth task involves assessing whether hallucinations are part of psychotic states and, if not, whether there are any pointers to why this symptom is presenting in this child at this particular time, including possible vulnerabilities and stressors in addition to other psychiatric morbidity. This assessment process is not always straightforward. In younger children or those with intellectual disability or in mistrustful and uncommunicative young people, it may require careful questioning by clinicians experienced in both interviewing children and diagnosing psychotic and other psychiatric states.

\section{The detailed exploration of the symptom}

Hallucinatory experiences can affect any of the five sensory modalities (Table 2). A comprehensive

\section{TABLE 2 Hallucinatory experiences in five sensory modalities: description and differential diagnosis}

\begin{tabular}{|c|c|c|}
\hline Sensory modality & Hallucinatory modality & Differential diagnosis \\
\hline Hearing & $\begin{array}{l}\text { Auditory hallucinations are the perception of sound in the absence } \\
\text { of external stimuli. These include a broad range of auditory } \\
\text { experiences, including hearing noises, melodies, ringing, whistling, } \\
\text { whispering, animal sounds and human voices. These can be either } \\
\text { friendly or distressing; voices can speak single words or elaborate } \\
\text { sentences, and can be commanding, insulting or commenting. }\end{array}$ & $\begin{array}{l}\text { They can be present in neurological conditions (e.g. temporal lobe } \\
\text { epilepsy) and a broad range of psychiatric disorders (including } \\
\text { schizophrenia and mood disorders). Differential diagnosis is } \\
\text { required with obsessional thoughts and post-traumatic auditory } \\
\text { flashbacks. }\end{array}$ \\
\hline Seeing & $\begin{array}{l}\text { Visual hallucinations are the perception of visual images in the } \\
\text { absence of external stimuli. They may range from simple visual } \\
\text { hallucinations or non-formed visual hallucinations (e.g. lights, } \\
\text { colours, shapes or incomplete objects) to complex or formed visual } \\
\text { hallucinations (e.g. elaborate vivid images of people, animals, } \\
\text { objects or scenarios). }\end{array}$ & $\begin{array}{l}\text { They can be present in neurological conditions (especially } \\
\text { migraine, occipital lobe tumour, neoplasms of optic nerve or retina), } \\
\text { substance misuse or medication side-effects (e.g. ranitidine, } \\
\text { fentanyl), but also in a range of psychiatric disorders (including } \\
\text { schizophrenia). Differential diagnosis is required with hypnagogic } \\
\text { or hypnopompic images, imaginary companions, intrusive images } \\
\text { in obsessive-compulsive disorder and post-traumatic visual } \\
\text { flashbacks. }\end{array}$ \\
\hline Sensing & $\begin{array}{l}\text { Tactile hallucinations involve the experience of tactile sensing in } \\
\text { the absence of external stimuli. They include experiences, e.g. of } \\
\text { being touched, held, hurt, burned or pushed. }\end{array}$ & $\begin{array}{l}\text { They can be present in substance misuse such as cocaine } \\
\text { consumption (Magnan syndrome - the sensation of insects } \\
\text { crawling underneath the skin), alcohol withdrawal or cannabis } \\
\text { use, medical conditions (peripheral neuropathy, high fever, Lyme } \\
\text { disease, skin cancer) or psychiatric disorders such as schizophrenia. }\end{array}$ \\
\hline Smelling & $\begin{array}{l}\text { Olfactory hallucinations involve smelling odours that are not really } \\
\text { present, e.g. unpleasant smells such as rotting flesh, vomit, urine, } \\
\text { faeces or smoke. }\end{array}$ & $\begin{array}{l}\text { They can be present in neurological conditions (i.e. temporal } \\
\text { lobe pathology, complex partial seizures or olfactory tumour) or } \\
\text { psychiatric conditions (e.g. schizophrenia or depression). }\end{array}$ \\
\hline Tasting & $\begin{array}{l}\text { Gustatory hallucinations are the perception of taste without a } \\
\text { stimulus. They tend to be strange or unpleasant tastes. }\end{array}$ & $\begin{array}{l}\text { They can be present in neurological conditions (i.e. temporal lobe } \\
\text { epilepsy) medication side-effects (e.g. methylphenidate), substance } \\
\text { misuse and psychiatric conditions (e.g. psychotic disorder). }\end{array}$ \\
\hline
\end{tabular}

Sources: de Leede-Smith \& Barkus (2013); Jardri et al (2014); Waters et al (2014). 
assessment requires detailed exploration of the symptom characteristics for each individual (Table 3). As a general rule, it is better to ask children and adolescents open questions where they can describe the experiences, or to provide a choice of answers from which they can choose, rather than closed questions that may guide and contaminate their answers. Descriptions of symptoms should be factual, avoiding florid interpretation.

In children, hallucinations are regarded as having clinical significance when: (i) they occur without clouding of consciousness, and (ii) they are either comparatively complex (e.g. voices containing a narrative or that are multiple, elaborate visions, multisensory experiences), or are distressing and impairing, or are linked to physical illness and/or active psychopathology and/or suicidality. Adult studies have identified markers of risk in the case of auditory verbal hallucinations. These include emotional valence driven by negative content, appraisal of the identity, intent and power of voices as a source of distress, perceived lack of control and presence of command hallucinations that may encourage harm to self or others (de LeedeSmith 2013). However, these findings are yet to be replicated in child and adolescent populations.

\section{Clinical scales to assist the assessment of hallucinations}

A number of clinician-rated scales have been used to assess the severity of hallucinations in adults with psychotic disorders (e.g. the Positive and Negative Syndrome Scale (PANSS; Kay 1987)) and these may be useful to quantify treatment progress in young people with psychoses.

\section{TABLE 3 The detailed exploration of hallucinations}

\begin{tabular}{|c|c|}
\hline Domain & Description \\
\hline Content & $\begin{array}{l}\text { The child should be asked to describe the content of their hallucinations - noises or voices, visual } \\
\text { images, smells, tactile sensations, tastes. In children with psychiatric disorders, hearing voices } \\
\text { addressing the child or talking among themselves or mixed auditory and visual hallucinations are } \\
\text { most commonly reported; visual perceptual anomalies are commonly part of confusional states. }\end{array}$ \\
\hline Timing & $\begin{array}{l}\text { Do they occur only in drowsiness? Are they part of a confusional state, or in the context of high } \\
\text { fever in young children? }\end{array}$ \\
\hline Frequency and duration & Experiences usually have clinical significance when frequent and/or continuous. \\
\hline $\begin{array}{l}\text { Belief/awareness of } \\
\text { reality }\end{array}$ & $\begin{array}{l}\text { This is difficult to explore with children: even children without psychotic disorders can be uncertain } \\
\text { as to whether voices are happening in the real world or are a product of their 'imagination' or mind. }\end{array}$ \\
\hline Level of distress & $\begin{array}{l}\text { Subjective report of the level of distress caused by the experiences, which may be related to } \\
\text { negative content (such as blaming voices) or level of intrusiveness. } \\
\text { Unpleasant hallucinatory experiences that interfere with the young person's activity and thoughts } \\
\text { are highly clinically significant and may be the driver to help-seeking. }\end{array}$ \\
\hline Observed behaviour & $\begin{array}{l}\text { This is particularly important in children under } 7 \text { and may be the only way of establishing the } \\
\text { presence of hallucinations. The same applies to older children with hostile or suspicious attitudes, } \\
\text { unwilling to share their unusual experiences with others. } \\
\text { Possible indicators are: listening into space, abrupt interference with speech, tendency to lateral } \\
\text { gazing with signs of hypervigilance, escaping behaviour, compliance with commanding messages, } \\
\text { searching for cameras/microphones, talking to themselves, inappropriate laughing, gesturing } \\
\text { towards empty space. }\end{array}$ \\
\hline Level of impairment & $\begin{array}{l}\text { Whether the young person continues with daily life activities (e.g. school, family life, pleasure and } \\
\text { social life) is a crucial indicator of clinical significance. }\end{array}$ \\
\hline Sociocultural factors & $\begin{array}{l}\text { Cultural beliefs in relation to these experiences may be relevant; e.g. in certain cultures, } \\
\text { hallucinations may become be mixed up with beliefs of possession by evil forces. }\end{array}$ \\
\hline Change over time & $\begin{array}{l}\text { The phenomenological features of hallucinations may fluctuate over time, possibly reflecting } \\
\text { changes in clinical state. }\end{array}$ \\
\hline \multicolumn{2}{|c|}{ Additional exploration for auditory verbal hallucinations } \\
\hline Inner/outer locus & $\begin{array}{l}\text { Auditory verbal hallucinations may be experienced as coming from inside the head or outside } \\
\text { the head (or both). It is important to know that children and young people may find it difficult to } \\
\text { make this distinction, and moreover that research suggests that the perceived location of these } \\
\text { experiences does not have any clear meaningful relationship with diagnostic or prognostic factors. }\end{array}$ \\
\hline Content & $\begin{array}{l}\text { They may be positive, neutral or negative. Negative voices (e.g. shaming themes, personal insults) } \\
\text { tend to be reported more in association with mood changes and mood disorders. Command voices } \\
\text { may result in risky behaviour. }\end{array}$ \\
\hline $\begin{array}{l}\text { Personification and } \\
\text { attributions }\end{array}$ & $\begin{array}{l}\text { Individuals often personify their voices. Young people may report knowing the identity of their } \\
\text { voices, and the voices may tell them their name. Attributions may be to the self ('I hear my own } \\
\text { voice'), to others ('I hear someone else talking to me') or to no specific source. }\end{array}$ \\
\hline
\end{tabular}

Sources: de Leede-Smith \& Barkus (2013); Waters et al (2014). 
More recently validated instruments explore the characteristics of psychotic symptoms regardless of diagnosis. For example, the Psychotic Symptoms Rating Scales (PSYRATS, 17 items; Haddock 1999) quantifies in considerable detail the multidimensional features of hallucinations (frequency, duration, location, loudness, origin, negativity, distress, disruption and controllability) and delusions, and has been validated in clinical and non-clinical samples; the Questionnaire for Psychotic Experiences (QPE, 50 items; Nijboer 2015) measures the presence, severity and phenomenological characteristics of auditory, visual and other sensory hallucinations and delusions. The PSYRATS is increasingly being used clinically as a measure of symptom change, whereas the QPE is a promising clinical scale at the earliest stages of research and clinical development. However, although these scales look promising, there is as yet little evidence on their use with children and young people.

\section{Differential diagnosis}

\section{Hallucinations and paediatric organic conditions}

A variety of paediatric organic conditions need to be considered and investigated when there are relevant clinical indicators based on detailed medical history and physical exploration. Freudenreich (2009) and Algon (2012) have outlined broad and specific screening for medical disorders in first-episode psychosis (Table 4).

\section{Hallucinations and paediatric psychiatric conditions}

As important as carefully exploring the nature of hallucinatory experiences is to assess the constellation of symptoms of which hallucinatory experiences form part for each particular child or adolescent. The psychiatric differential diagnosis of hallucinations includes:

- psychiatric disorders where psychotic symptoms are a hallmark (hereafter: psychotic disorders), as in schizophrenia spectrum (i.e. schizophrenia, schizoaffective, schizotypal, acute and transient psychotic disorders) and mood disorders (i.e. bipolar disorder and major depression with psychotic symptoms); each of these requires specific symptom combinations and time frames to reach clinical diagnosis level (World Health Organization 1992); for an initial psychotic presentation, all these disorders are grouped into first-episode psychosis

- psychiatric disorders where hallucinations are not a hallmark but an associated symptom, e.g. emotional and behavioural disorders
TABLE 4 Paediatric organic conditions that may present with hallucinations or related perceptual phenomena - with or without reduced levels of consciousness

\begin{tabular}{|c|c|}
\hline Neurological disorders & $\begin{array}{l}\text { Migraines with aura (e.g. Alice in Wonderland syndrome), sleep } \\
\text { disorders (e.g. narcolepsy), epilepsy (e.g. complex partial seizures, } \\
\text { temporal lobe epilepsy), malignancies, brain-occupying lesions, } \\
\text { head injury, demyelinating diseases (multiple sclerosis), basal } \\
\text { ganglia disease (Wilson disease, Huntington disease and Fahr } \\
\text { disease), delirium }\end{array}$ \\
\hline Infections & $\begin{array}{l}\text { Meningitis, encephalitis, or febrile illness; HIV infection. } \\
\text { Travellers should consider cerebral malaria, toxoplasmosis, } \\
\text { neurocysticercosis and sleeping sickness }\end{array}$ \\
\hline Autoimmune disorders & $\begin{array}{l}\text { Systemic lupus erythematosus, Hashimoto encephalopathy, } \\
N \text {-methyl-D-aspartate (NMDA) encephalitis, paraneoplastic limbic } \\
\text { encephalitis }\end{array}$ \\
\hline Endocrine diseases & $\begin{array}{l}\text { Thyroid (hyperthyroidism or hypothyroidism) and parathyroid } \\
\text { disease, adrenal disease, steroid-producing tumours located } \\
\text { in either the adrenal gland (Cushing disease) or other tissues } \\
\text { (eg. ectopic Cushing syndrome from small-cell lung cancer), } \\
\text { insulinomas, pheochromocytoma }\end{array}$ \\
\hline Metabolic disorders & $\begin{array}{l}\text { Acute intermittent porphyria, Tay-Sachs disease, Niemann-Pick } \\
\text { disease type C, hypoglycaemia, electrolyte imbalance (calcium, } \\
\text { phosphate, sodium and magnesium derangements), intoxication } \\
\text { with lead and copper }\end{array}$ \\
\hline Nutritional deficiencies & Vitamin $B_{12}$ deficiency, thiamin deficiency, niacin deficiency \\
\hline Congenital disorders & $\begin{array}{l}\text { More than } 60 \text { congenital disorders, including genetic and } \\
\text { metabolic disorders and lysosomal storage diseases }\end{array}$ \\
\hline Substance misuse & $\begin{array}{l}\text { Cannabis, lysergic acid diethylamide (LSD), cocaine, } \\
\text { amphetamines, ecstasy (3,4-methylenedioxymethamphetamine), } \\
\text { opiates and barbiturates, PCP ('angel dust' or phencyclidine), } \\
\text { alcohol misuse or withdrawal }\end{array}$ \\
\hline Medication toxicity & $\begin{array}{l}\text { Stimulants (i.e. methylphenidate), anticholinergics, corticosteroids, } \\
\text { beta-blockers (i.e. metoprolol),digitalis, dopaminergic agonists, } \\
\text { interferon, antituberculous drugs (e.g. isoniazid), antibiotics } \\
\text { (ciprofloxacin), antivirals (HIV medications), anticonvulsants, } \\
\text { antineoplastics, pain medications (opioids), and others }\end{array}$ \\
\hline
\end{tabular}

Sources: Freudenreich et al, 2009; Algon et al, 2012.

- psychiatric disorders with psychopathological features which may resemble those of hallucinations, such as intrusive obsessions in obsessivecompulsive disorder, intrusive images/thoughts in post-traumatic stress disorder, or elaborate magical bizarre thoughts in autism spectrum disorder.

\section{Hallucinations and psychotic disorders}

First-episode psychosis is defined as more than a week of unremitting frank psychotic symptoms, namely clinically defined delusions, hallucinations, passivity experiences or severe thought disorder. Psychotic symptoms are considered frank if they score higher than 4 (moderate) on the PANSS (the scale runs from 1 to 7 : absent to extreme). In psychotic states, hallucinations co-occur with a constellation of symptoms, as part of the clinical psychosis phenotype described by five psychopathological dimensions (Dikeos 2006):

- the positive dimension: delusions, ideas of reference, unusual thought content, hallucinations, grandiosity and suspiciousness/persecution 
- the negative dimension: alogia, affective flattening, avolition, apathy, anhedonia, asociality, social withdrawal, stereotyped thinking and motor retardation

- the disorganised dimension: conceptual disorganisation, positive formal thought disorder, difficulty in abstract thinking, derailment, tangentiality, incoherence, illogicality, circumstantiality, associative loosening, inattention/distractibility, disorientation, inappropriate affect, bizarre behaviour, mannerisms and posturing

- the depressive dimension: observed depression, hopelessness, self-deprecation, feelings of guilt, guilty ideas of reference, early wakening, suicidal ideation, anxiety and active social avoidance

- the manic/excitement dimension: pressured speech, excessive activity, racing thoughts, elevated mood, increased sociability, reduced need for sleep, reckless activity, irritable mood, increased self-esteem and grandiose delusions.

An individual can score high or low on the different dimensions, which may coexist. Close monitoring over time will be required to observe the direction in which psychopathology evolves. It may veer towards a schizophrenia spectrum or mood disorder picture.

\section{Characteristics of hallucinations in psychotic disorders}

A retrospective study of hallucinations in children and adolescents referred to the Maudsley Hospital in London identified auditory hallucinations as the most common psychotic symptom in those with psychotic disorders (schizophrenia, manic-depressive illness, schizoaffective or unspecified psychoses), present in three-quarters of the sample, and it described their clinical manifestations (Garralda 1984b, 1985). In terms of content, voices tended to tell the children to carry out actions which, in half the cases, could be considered as wrongdoings. Some contained suicidal instigations, and occasionally there was a direct relationship with suicidal symptoms. Their tone was equally likely to be unpleasant as pleasant, and the voices were varyingly attributed to unspecified or familiar people such as school contacts or parents; more rarely they were thought to be God. Some children interpreted the voices as their own thoughts being spoken aloud or other people speaking their thoughts. Visual hallucinations were less common, variously described as of unidentified people or family members, animals or objects; rarely would a child report seeing the devil.

Hallucinations co-occurred with delusional beliefs (mainly of a paranoid or persecutory nature, such as complaining that people were poisoning or conspiring against them), abnormalities in language production (incoherence, mutism or laconism, repetitive speech), inappropriate affect (including giggling), bizarre behaviour, hypoactivity and social withdrawal. Schneiderian first-rank symptoms were rare, present in $15 \%$ of the sample.

The presence of hallucinations in this sample (when compared with children with psychoses who did not report hallucinations) was linked to reporting precipitants of illness, symptoms of depression and anxiety, and problems with reading (Garralda 1984b), suggesting a role of stressors, mood changes and cognitive vulnerability in the experience of hallucinations in children and young people with psychotic disorders.

\section{Psychotic schizophrenia spectrum disorders}

Youth-onset schizophrenia is an infrequent but important cause of hallucinations in young people. The prevalence of schizophrenia increases rapidly from age 14 and accounts for $24 \%$ of UK psychiatric admissions of young people aged 10-18 (National Institute for Health and Care Excellence (NICE) 2013). Schizophrenia spectrum disorders are commonly preceded by the so-called prodromal period, lasting up to 12 months, in which the child or young person's behaviour and experience are altered, leading to social withdrawal or impaired functioning. In the especially rare prepubertal or childhood-onset schizophrenia (David 2011), auditory and visual hallucinations have been documented in 95\% and $80 \%$ of children respectively during their hospital admissions. In this group, visual hallucinations showed a significant relationship with lower IQ and earlier age at onset, and tactile (60\%) and olfactory (30\%) hallucinations were observed only in children with visual hallucinations.

\section{Psychotic mood disorders}

Mood changes are a particularly common association of hallucinations in children and should therefore be explored carefully. Both extremes of mood disorder (i.e. the manic phase of bipolar disorder as well as the depressive phase of major depression) may present with psychotic hallucinatory experiences, which tend to be mood congruent. In paediatric populations with bipolar disorder (Kowatch 2005), increased energy, distractibility and pressured speech, followed by racing thoughts, decreased need for sleep and poor judgement, are the most common symptoms of mania, in contrast to flight of ideas and hypersexuality, which are less frequently 
present. It is of note that irritability and dysphoria are not distinctive features of mania, as they may be the predominant mood state in young people presenting with the constellation symptomatology of depression (i.e. anhedonia, fatigue and suicidal ideation) (Chang 2009). In a sample of 257 children and adolescents with bipolar I disorder (Tillman 2008), excluding the perceptual experiences of imaginary companions or voices calling their name (which occurred in $43.6 \%$ of cases), visual hallucinations were the most frequent hallucination type (16\%), followed by command auditory hallucinations (15.6\%), tactile (10.1\%) and olfactory $(8.2 \%)$ hallucinations, and conversing voices $(7.4 \%)$, commenting voices $(6.6 \%)$ and other verbal hallucinations (5.4\%).

\section{Hallucinations and non-psychotic psychiatric disorders}

Population studies show that children who report hallucinatory experiences score significantly higher than those who do not on general and specific measures of psychopathology, including both internalising and externalising features (Scott 2009; Kelleher 2012b). This has been replicated in clinical samples, where hallucinations co-occur in children and young people presenting with anxiety disorders, stress reactions and conduct/oppositional disorders (Garralda 1984a; Askenazy 2007; Braakman 2009). Hallucinations are, however, not a frequent feature of clinical presentations: in Ulloa et al's (2000) study of children and adolescents referred to a mood and anxiety disorders clinic, psychotic symptoms (mainly hallucinations) were reported by $4 \%$ and suspected in a further 4\%: definite symptoms were associated with mood disorders and suicidal ideation, the latter mediated by the presence of a mood disorder.

There has been comparatively little work to characterise hallucinations in children with emotional and behavioural disorders (Ulloa et al looked only at mood disorders), but the above-mentioned paediatric hallucinations study at the Maudsley Hospital described in some detail the experiences of a group of children with hallucinations in the context of emotional and conduct disorders (Garralda 1984a). As in the children with psychotic states, auditory hallucinations predominated, and visual phenomena were reported by about half. The length of time hallucinations had been present ranged from 1 week to several years, and episodes tended to be brought on by stressful events, distress, naughtiness or temper. Three-quarters of the children reported that voices addressed them.
In half of these cases the voices asked them to do something wrong and unpleasant, and in over a third they made threatening comments. About half of the children said that they were anxious and frightened during these experiences, and a quarter said they fought the voices, refusing to obey orders. There was a trend for the voices to be located predominantly in the child's internal space before the age of 13 and in external space after that age. Visual hallucinations included seeing frightening objects such as skeletons or ghosts, but a number of children also talked about seeing recently deceased people.

The children with hallucinations and nonpsychotic diagnoses were found to be older and with lower IQ than the majority of clinic attenders and they were also more likely to be admitted as inpatients, a proxy for illness severity. In comparison with a matched control group of children without hallucinations, they were found to have experienced more stressors precipitating illness, and they displayed more mood changes, and dissociative symptoms (e.g. derealisation) and episodes, as well as more neurodevelopmental deficits (e.g. problems with reading, and discrepancies in verbal and performance IQ). Hallucinations were not linked to lower socioeconomic backgrounds, social deprivation, sensory deficits, vivid imagination or daydreaming. At long-term follow-up in adulthood (Garralda 1984c), the psychiatric adjustment of children with a history of hallucinations was comparable with that of controls, but although rates of psychoses were not increased among these children, they continued to be more likely to experience complex hallucinations and dissociative phenomena (e.g. depersonalisation and derealisation, déjà vu and short dissociative episodes) over the years.

These findings are in keeping with the increasing evidence of: first, a relationship between early traumatic events and stress reactions, including hallucinations (Braakman 2009) and in a minority of individuals psychotic disorders (de Leede-Smith 2013); and, second, the possibility of dissociation as a mediator in the relationship between childhood trauma and hallucination proneness (Varese 2012). Links between hallucinations and dissociation, low intellectual functioning and emotional dysregulation have since been confirmed by epidemiological findings and clinical reports (Linscott 2013; Jadri 2014). This points to the relevance of emotional dysregulation and its perceptual correlates in young people's responses to traumatic events. For the clinician, the findings indicate the advisability of exploring the presence of possibly unacknowledged traumas and losses, as well as dissociative tendencies and cognitive 
vulnerabilities in these children, in addition to assessing mood regulation and suicidality.

There is little information on the short-term outcome of hallucinations in clinical samples. In a small group of 13 prepubertal out-patients with hallucinatory experiences, half were free of these 3 months following assessment and at 1-year followup, about 1 in 10 had recovered but had had a relapse following new stresses, and the rest (under one-third) continued to report hallucinatory experiences (Askenazy 2007).

\section{Schizotypal disorder and autism spectrum presentations}

Psychotic-like features such as hallucinations pose a further challenge to clinicians when they occur in the context of either an autism spectrum or a schizotypal disorder. Schizotypal disorder is characterised by eccentric behaviour and anomalies of thinking and affect that resemble those seen in schizophrenia, and may include unusual perceptual experiences, as well as transient quasi-psychotic episodes with intense hallucinations. Schizotypal disorder is rarely diagnosed in children, but in adolescents it may overlap with autistic disorders (Barneveld 2011) and account for psychotic symptoms in some adolescents with autism. The differentiation of transient quasi-psychotic states from full-blown schizophrenic disorder may require particularly careful assessment and observation over time in young people with autism and hallucinations.

\section{Management plans}

Childhood hallucinatory experiences in nonclinical samples rarely cause substantial suffering in children's lives, and high rates of symptom discontinuation make treatment usually unnecessary. In clinical samples of children presenting with psychotic disorders, specific treatment strategies will be put into place, while in those with non-psychotic disorders, treatment of the associated psychiatric disorder and/or therapeutic work aimed at stress/anxiety reduction and mood regulation may be expected to improve hallucination-related distress. Occasionally, and in the face of persisting hallucinations that do not respond to appropriate interventions, antipsychotic medication may be helpful (Vickers 2002).

\section{Psychotic disorders}

When hallucinations are part of a first psychotic episode, early identification and treatment are indicated. NICE guidelines recommend urgent specialist referral to either child and adolescent mental health services (CAMHS) or an early intervention in psychosis service (NICE 2013). The currently recommended treatment includes the use of antipsychotic medication (risperidone or aripiprazole, for at least 12 months) in conjunction with psychological interventions, namely psychoeducation, discontinuation of illicit drug use, reduction of stresses, family intervention and individual cognitive-behavioural therapy. Duration of untreated psychosis (DUP), which refers to the time between the onset of psychotic symptoms continuous with the presenting episode and the initiation of continuous ( $>30$ days) antipsychotic medication, has become established as an important factor associated with outcome: the longer the DUP, the worse the subsequent psychosis, so early diagnosis and treatment are called for.

Antipsychotic medication is not recommended for psychotic symptoms that are not sufficient for a diagnosis of first-episode psychosis, nor should it be used with the aim of decreasing the risk of psychosis (NICE 2013). However, young people with an at-risk mental state should be carefully monitored for signs of transition to frank psychosis.

\section{Non-psychotic presentations}

When hallucinations are part of anxiety, posttraumatic stress, disruptive or substance misuse disorders, treatment of the disorder is indicated. The hallucinations themselves deserve particular attention and monitoring if they are complex (e.g. containing a narrative, involving multiple voices, multisensory), distressing and impairing even after the accompanying psychopathology has subsided or if it does not respond to treatment.

\section{Psychological therapy for hallucinations}

Since distress is one of the risk markers for hallucinations, stress management can be a major target of specific psychological therapies. In addition, psychological theories of auditory hallucinations have been developed over the years, with related therapeutic modalities (Thomas 2014).

Cognitive-behavioural therapy (CBT) for psychotic symptoms has been evaluated as an adjunct to routine care in the context of psychotic states; results suggest a modest reduction in symptom severity, but there have been few trials focusing on hallucinations themselves. Recent developments in the management of hallucinations include mindfulness-based therapies, competitive memory training and compassionate mind training, which aim to promote greater resilience to critical commenting. Another recent direction has been the use of computer-generated avatars that enable the therapist to role-play the voice 
to help the person practise different responses to the experience. These techniques ${ }^{\dagger}$ have been used primarily with adults and require further evaluation, and their suitability and efficacy for children are not known.

Edelsohn (2006) described a modification of CBT as a brief intervention for hallucinations in children presenting to psychiatric emergency services. It consists of gaining an appreciation of the child's beliefs about the hallucinations ('What do they mean?'), details of how they started, and whether the child can start or stop them, followed by helping the child identify alternative explanations for the hallucinations and introducing coping strategies.

\section{Psychoeducation when hallucinations are part of non-psychotic disorders}

If hallucinations present in the context of nonpsychotic disorders, psychoeducation for the child and family about the symptoms is important. The popular understanding of hallucinations is that they are an expression of severe mental illness. The symptoms can therefore be very alarming, which may partly explain why they tend to present as psychiatric emergencies (Edelsohn 2006). This may be compounded by concern arising because of a family history of psychotic illness. It is therefore highly relevant for the clinician to carry out a careful differential diagnosis and share this with the family, explaining the potential presence of hallucinations across a number of child psychiatric diagnoses and their response to treatment and conveying information about the limited predictive value of childhood hallucinatory experiences for adult psychotic states.

Destigmatisation and normalisation, alongside attention to concurrent stressors and psychiatric disorders (including mood disorders and suicidality as well as any concurrent neurodevelopmental risks), are thus key elements of therapeutic input.

\section{References}

Algon S, Yi J, Calkins ME, Kohler C, et al (2012) Evaluation and treatment of children and adolescents with psychotic symptoms. Current Psychiatry Reports, 14: 101-10.

Askenazy FL, Lestideau K, Meynadier A, et al (2007) Auditory hallucinations in pre-pubertal children: a one-year follow-up, preliminary findings. European Child \& Adolescent Psychiatry, 16: 411-5.

Barneveld PS, Pieterse J, de Sonneville L, et al (2011) Overlap of autistic and schizotypal traits in adolescents with autism spectrum disorders. Schizophrenia Research, 126: 231-6.

Braakman MH, Kortmann FA, van den Brink W (2009) Validity of 'posttraumatic stress disorder with secondary psychotic features': a review of the evidence. Acta Psychiatrica Scandinavica, 119: 15-24.

Casey P, Brendan K (2007) Fish's Clinical Psychopathology: Signs and Symptoms in Psychiatry (3rd edn). Gaskell.
Chang K (2009) Challenges in the diagnosis and treatment of pediatric bipolar depression. Dialogues in Clinical Neuroscience, 11: 73-80.

David CN, Greenstein D, Clasen L, et al (2011) Childhood onset schizophrenia: high rate of visual hallucinations. Journal of the American Academy of Child and Adolescent Psychiatry, 50: 681-6.

de Leede-Smith S, Barkus E (2013) A comprehensive review of auditory verbal hallucinations: lifetime prevalence, correlates and mechanisms in healthy and clinical individuals. Frontiers in Human Neuroscience, 7: 367.

Despert JL (1948) Delusional and hallucinatory experiences in children. American Journal of Psychiatry, 104: 528-37.

Dikeos DG, Wickham H, McDonald C, et al (2006) Distribution of symptom dimensions across Kraepelinian divisions. British Journal of Psychiatry, 189: 346-53

Dominguez MDG, Saka MC, Lieb R, et al (2010) Early expression of negative/disorganized symptoms predicting psychotic experiences and subsequent clinical psychosis: a 10-year study. American Journal of Psychiatry, 167: 1075-82.

Dominguez MDG, Wichers M, Lieb R, et al (2011) Evidence that the onset of clinical psychosis is an outcome of progressively more persistent subclinical psychotic experiences: an 8-year cohort study. Schizophrenia Bulletin, 37: 84-93.

Edelsohn GA (2006) Hallucinations in children and adolescents: considerations in the emergency setting. American Journal of Psychiatry, 163: 781-5.

Freudenreich 0, Schulz SC, Goff DC (2009) Initial medical work-up of first-episode psychosis: a conceptual review. Early Intervention in Psychiatry, 3: 10-18

Fusar-Poli P, Bechdolf A, Taylor MJ, et al (2013) At risk for schizophrenic or affective psychoses? A meta-analysis of DSM/ICD diagnostic outcomes in individuals at high clinical risk. Schizophrenia Bulletin, 39: 923-32.

Garralda ME (1984a) Hallucinations in children with conduct and emotional disorders: I. The clinical phenomena. Psychological Medicine, 14: 589-96.

Garralda ME (1984b) Psychotic children with hallucinations. British Journal of Psychiatry, 145: 74-7.

Garralda ME (1984c) Hallucinations in children with conduct and emotional disorders: II. The follow-up study. Psychological Medicine, 14: $597-604$

Garralda ME (1985) Characteristics of the psychoses of late onset in children and adolescents: a comparative study of hallucinating children. Journal of Adolescence, 8: 195-207.

Haddock G, McCarron J, Tarrier N, et al (1999) Scales to measure dimensions of hallucinations and delusions: the psychotic symptom rating scales (PSYRATS). Psychological Medicine, 29: 879-89.

Jardri R, Bartels-Velthuis AA, Debbane M, et al (2014) From phenomenology to neurophysiological understanding of hallucinations in children and adolescents. Schizophrenia Bulletin, 40 (suppl 4): S221-32.

Kay SR, Fiszbein A, Opler LA (1987) The Positive and Negative Syndrome Scale (PANSS) for schizophrenia. Schizophrenia Bulletin, 13: $261-76$.

Kelleher I, Connor D, Clarke MC, et al (2012a) Prevalence of psychotic symptoms in childhood and adolescence: a systematic review and meta-analysis of population-based studies. Psychological Medicine, 42: 1857-63

Kelleher I, Keeley H, Corcoran P, et al (2012b) Clinicopathological significance of psychotic experiences in non-psychotic young people: evidence from four population-based studies. British Journal of Psychiatry, 201: 26-32.

Kowatch RA, Youngstrom EA, Danielyan A, et al (2005) Review and meta-analysis of the phenomenology and clinical characteristics of mania in children and adolescents. Bipolar Disorders, 7: 483-96.
†Turkington et al discuss coping strategies for auditory hallucinations on pp. 391-396, this issue. In the previous issue (22 (5): $301-310$ ) 0 'Hanlon et al examined the therapeutic use of technology for psychosis. Ed.
MCQ answers

$1 \mathrm{c} \quad 2 \mathrm{~d} \quad 3 \mathrm{~b} \quad 4 d \quad 5$ b 
Linscott RJ, van Os J (2013) An updated and conservative systematic review and meta-analysis of epidemiological evidence on psychotic experiences in children and adults: on the pathway from proneness to persistence to dimensional expression across mental disorders. Psychological Medicine, 43: 1133-49.

National Institute for Health and Care Excellence (2013) Psychosis and Schizophrenia in Children and Young People: Recognition and Management (Clinical Guideline CG155). NICE.

Nijboer M (2015) Validating the Questionnaire for Psychotic Experiences (Masters Thesis). Faculty of Social and Behavioural Sciences, University of Utrecht.

Pearson D, Rouse H, Doswell S, et al (2001) Prevalence of imaginary companions in a normal child population. Child: Care, Health and Development, 27: 13-22.

Piaget J (1932) Le jugement moral chez l'enfant. Reprinted (1997) as The Moral Judgment of the Child (trans M. Gabian). Free Press Paperbacks.

Scott J, Martin G, Bor W, et al (2009) The prevalence and correlates of hallucinations in Australian adolescents: results from a national survey. Schizophrenia Research, 107: 179-85.

Shears D, Elison S, Garralda ME, et al (2005) Near-death experiences with meningococcal disease. Journal of the American Academy of Child and Adolescent Psychiatry, 44: 630-1.

Smeets F, Lataster T, Dominguez MDG, et al (2012) Evidence that onset of psychosis in the population reflects early hallucinatory experiences that through environmental risks and affective dysregulation become complicated by delusions. Schizophrenia Bulletin, 38: 531-42.

Smith RA, Wright B, Bennett S (2015) Hallucinations and illusions in migraine in children and the Alice in Wonderland Syndrome. Archives of Disease in Childhood, 100: 296-28.
Thapar A, Heron J, Jones RB, et al (2012) Trajectories of change in self-reported psychotic-like experiences in childhood and adolescence. Schizophrenia Research, 140: 104-9.

Thomas N, Hayward M, Peters E, et al (2014) Psychological therapies for auditory hallucinations (voices): current status and key directions for future research. Schizophrenia Bulletin, 40 (suppl 4): S202-12.

Tillman R, Geller B, Klages T, et al (2008) Psychotic phenomena in 257 young children and adolescents with bipolar I disorder: delusions and hallucinations (benign and pathological). Bipolar Disorders, 10: 45-55.

Ulloa RE, Birmaher B, Axelson D, et al (2000) Psychosis in a pediatric mood and anxiety disorders clinic: phenomenology and correlates. Journal of the American Academy of Child and Adolescent Psychiatry, 39: $337-45$

van Rossum I, Dominguez MDG, Lieb R, et al (2011) Affective dysregulation and reality distortion: a 10 -year prospective study of their association and clinical relevance. Schizophrenia Bulletin, 37: 561-71.

Varese F, Barkus E, Bentall RP (2012) Dissociation mediates the relationship between childhood trauma and hallucination-proneness. Psychological Medicine, 42: 1025-36.

Vickers B (2002) Case study: the treatment of auditory hallucinations in children with emotional disorders. Child and Adolescent Mental Health, 7: $25-30$.

Waters F, Collerton D, Ffytche DH, et al (2014) Visual hallucinations in the psychosis spectrum and comparative information from neurodegenerative disorders and eye disease. Schizophrenia Bulletin, 40 (supp| 4): S233-45.

World Health Organization (1992) The ICD-10 Classification of Mental and Behavioural Disorders: Clinical Descriptions and Diagnostic Guidelines. WHO

\section{MCOs}

Select the single best option for each question stem

\section{The frequency of hallucinatory} experiences in children and young people in the general population is closest to:

\section{a $0.1 \%$}

b $1 \%$

c $10 \%$

d $20 \%$

e $30 \%$

2 The rate of discontinuation over time of hallucinatory experiences in children in the general population is closest to:

a $20 \%$

b $40 \%$

c $60 \%$

d $80 \%$

e $100 \%$.
3 The risk of a future psychotic state in children in the general population with psychotic symptoms/hallucinations is closest to:

a $1 \%$

b $10 \%$

c $20 \%$

d $30 \%$

e $40 \%$.

4 In clinical samples of children with nonpsychotic states, hallucinations have been found to be associated with:

a imaginary companions

b a rich fantasy life

c severe social deprivation

d dissociative experiences and emotional dysregulation

e parental psychotic states
5 The clinical management of hallucinations in children primarily involves:

a CBT for psychotic states

b treatment of the concurrent psychiatric disorder

c antipsychotic medication

$d$ reduction of the duration of untreated

psychosis

e mindfulness training. 